Revista de Psicología Vol. 37 (1), 2019 (ISSN 0254-9247)

\title{
Invarianza del Cuestionario de Dependencia Emocional entre sexos y situación sentimental en universitarios
}

\author{
Mariantonia Lemos ${ }^{1}$, Carolina Vásquez-Villegas ${ }^{2}$, Juan Pablo Román-Calderón ${ }^{3}$ \\ Universidad EAFIT, Colombia
}

El objetivo de este estudio fue evaluar la estructura factorial y la invarianza entre grupos del Cuestionario de Dependencia Emocional (CDE) mediante una muestra aleatoria de 569 universitarios, $67.8 \%$ mujeres y $51.2 \%$ con pareja. El Análisis Factorial Confirmatorio Multigrupo (AFCMG) mostró invarianza métrica para el CDE. Específicamente, los resultados indicaron que cinco de las seis escalas del cuestionario poseen varianzas extraídas promedio superiores al 53\% y una fiabilidad compuesta superior a .75. La escala de Expresión límite fue excluida porque no cumplió con el criterio de validez convergente. Se encontró que el CDE posee invarianza métrica fuerte entre sexos, $\chi^{2}(365)=750.14, p<.05$; RMSEA = .07; $\mathrm{CFI}=0.92 ; \mathrm{TLI}=.92 ; \mathrm{y}$ presencia o no de pareja, $\chi^{2}(365)=750.30, p<.05 ; \mathrm{RMSEA}=.06$; $\mathrm{CFI}=.92 ; \mathrm{TLI}=.92$.

Palabras clave: dependencia, diferencias entre sexos, parejas, modelos de ecuaciones estructurales, análisis factorial.

Invariance of the Emotional Dependence Questionnaire between sexes and relationship status in university students

This work aimed to explore the factorial structure and the measurement invariance between groups of the emotional dependence questionnaire (EDQ). Participants included a random sample of 569 university students, $67.8 \%$ women and $51.2 \%$ had a romantic partner. The multigroup confirmatory factor analysis (MG-CFA) showed metric invariance for the EDQ. Particularly, the findings indicate that five of six scales of the questionnaire have

1 Doctora en Psicología. Profesora titular del pregrado en psicología de la Universidad Eafit. Dirección postal: Universidad EAFIT. Cra 49 \#7 sur 50 Medellín, Colombia. Contacto: mlemosh@eafit.edu.com. ORCID: http://orcid.org/0000-0002-9131-4704

2 Estudiante de psicología de la Universidad Eafit. Dirección postal: Universidad EAFIT. Cra 49 \#7 sur 50, Medellín, Colombia. Contacto: cvasqu29@eafit.edu.com. ORCID: https:// orcid.org/0000-0002-1301-7333

3 Doctor en Psicología Organizacional. Profesor titular del Departamento de Negocios Internacionales de la Universidad Eafit. Dirección postal: Universidad EAFIT. Cra 49 \#7 sur 50, Medellín, Colombia. Contacto: jromanca@eafit.edu.com. ORCID: https://orcid. org/0000-0002-4352-8513 
an average variance extracted higher than $53 \%$ and a composite reliability of more than 0.75; the borderline expression scale was excluded. It was found that the EDQ has a strong measurement invariance degree between genders, $\chi^{2}(365)=750.14, p<.05$; RMSEA $=.07$; $\mathrm{CFI}=.92 ; \mathrm{TLI}=.92$; and the presence of a partner, $\chi^{2}(365)=750.30, p<.05$; $\mathrm{RMSEA}=.06$; $\mathrm{CFI}=.92 ; \mathrm{TLI}=.92$.

Keywords: dependence, gender differences, couples, structural equation modeling, factor analysis.

\section{Invariância do Questionário de Dependência Emocional entre sexos e situaçáo senti- mental em universitarios}

O objetivo foi avaliar a estrutura fatorial e a invariância entre os grupos do Questionário de Dependência Emocional (QMC) por meio de uma amostra aleatória de 569 universitários, 67.8\% mulheres e 51.2\% com casais. A Análise Fatorial Confirmatória Multigrupos (AFCMG) mostrou invariância métrica para o QDE. Especificamente, os resultados indicaram que cinco das seis escalas do questionário têm variâncias média extraídas superior a $53 \%$ e uma confiabilidade composta maior que .75. A escala de expressão limite foi excluída por não atender ao critério de validade convergente. Encontrou-se que o QDE possui forte invariância métrica entre os sexos, $\chi 2(365)=750.14$, p <.05; RMSEA= .07; CFI = .92; TLI= .92; e presença ou ausência de um casal, $\chi^{2}(365)=750.30, \mathrm{p}<.05$; RMSEA= .06 ; CFI= $.92 ; \mathrm{TLI}=.92$.

Palavras-chaves: dependência, diferenças de gênero, casais, modelos de equaçóes estruturais, análise fatorial.

L'invariance du Questionnaire de Dépendance Emotionnelle avec universitaires entre les sexes et la situation sentimentale dans universitaires

L'objectif c'était l'évaluation de la structure factorielle et l'invariance entre les groups du Questionnaire de Dépendance Emotionnelle (QDE) avec un échantillon aléatoire de 569 universitaires, $67.8 \%$ féminine et $51.2 \%$ en couple. L'analyse factoriel confirmatoire multi group (AFCMG) a montré invariance métrique pour le QDE. En particulier, les résultats indiquent que cinq des six échelles du questionnaire ont à moyenne variance extrait plus hautes que $53 \%$ et une fiabilité composée plus haute à 0.75 . L'échelle d'expression limite c'était exclu parce qu'elle n'avait pas rempli le critère de validité convergente. On a trouvé que le QDE a invariance métrique forte entre les sexes, $\chi^{2}(365)=750.14, p<.05$; RMSEA= .07 ; $\mathrm{CFI}=.92$; $\mathrm{TLI}=.92$; présence ou pas du couple, $\chi^{2}(365)=750.30, p<.05$; RMSEA $=$ $.06 ; \mathrm{CFI}=.92 ; \mathrm{TLI}=.92$.

Mots clés: dépendance, différence entre le genres, couples, modèle d'équations estructurelle, analyse factoriel 
La dependencia emocional se entiende como un patrón crónico de demandas afectivas insatisfechas que llevan a la persona a buscar desesperadamente satisfacerlas mediante relaciones interpersonales estrechas (Castello, 2005). Este concepto implica aspectos cognitivos, emocionales, motivacionales y comportamentales que toman al otro como fuente de satisfacción y seguridad, de tal forma que se concibe que la vida solo puede ser vivida en pareja, lo que genera malestar y limita el deterioro del desempeño autónomo de las personas (Antman, Selwyn, Braunwald \& Loscalzo, 2009; Cubas-Ferreyra, Espinoza-Rueckner, Galli-Cambiaso \& Terrones-Paredes, 2004; Hirschfeld et al., 1977; Izquierdo \& Gómez-Acosta, 2013).

Este concepto presenta asociaciones con conceptos como el apego preocupado, el cual está caracterizado por un estilo interpersonal en la adultez en el que las personas se muestran excesivamente dependientes de sus relaciones tanto para el apoyo emocional como tangible (Darcy, Davila \& Beck, 2005); sin embargo en la dependencia emocional las necesidades que se perciben como insatisfechas y que se pretenden llenar con otra persona son netamente emocionales (Castello, 2005). Además, es importante anotar qué modelos estructurales han evidenciado que la dependencia fuera un factor mediador entre el apego y la sintomatología emocional (Cantazaro \& Wei, 2010). Por otra parte, la sociotropía es otro concepto que se podría asociar con la dependencia emocional, en tanto hace referencia a la preocupación excesiva por obtener y mantener la aprobación y el soporte de otros. Sin embargo se ha propuesto que este factor formaría parte del constructo general de dependencia interpersonal o emocional (Bruch, Rivet, Heimberg, Hunt \& McIntosh, 1999). Finalmente, las personas con trastorno de personalidad dependiente presentan comúnmente necesidades afectivas insatisfechas y una visión de sí mismo como indefenso o con alta vulnerabilidad (Beck, Davis \& Freeman, 2015; Bornstein, 2006a). En la dependencia emocional la visión de sí mismo no está tan afectada, 
mientras que la visión de otros está marcada por la desconfianza (Lemos, Jaller, González, Díaz \& De la Ossa, 2012).

La dependencia emocional es una expresión extrema de la necesidad normal que tenemos como seres humanos de estar en contacto con otros, de tal manera que límites normales de esta están relacionados con calidez y valoración de las relaciones interpersonales (Bornstein et al., 2004; Castello, 2005; Dunkley, Blankstein, Zuroff, Lecce \& Hui, 2006; Nilakantan, Younger, Aron \& Mackey, 2014; Schulte, Mongrain \& Flora, 2008). Es por esto que la dependencia emocional es abordada desde un modelo dimensional donde se señala esta como un constructo similar al de la personalidad (Deary, Peter, Austin \& Gibson, 1998; Millon \& Everly, 1994; Roberts, Wasik, Casto \& Ramey, 1991; Tirapu, Perez, Calvo \& Mata, 2005), en tanto su manifestación dentro de unos límites adecuados es evidencia de salud mental, mientras su activación excesiva puede ser considerada como un factor de vulnerabilidad para el desarrollo de psicopatología. Al respecto, algunos estudios señalan su asociación con trastornos como la depresión (Bornstein, 2012; Davila, Steinberg, Kachadourian, Cobb \& Fincham, 2004; Sanathara, Gardner, Prescott \& Kendler, 2003), trastornos de personalidad (Bornstein, 1998; Morse, Robins \& Gittes-Fox, 2002), aspectos psicopatológicos en general (Santamaría et al., 2015); y, finalmente, se ha relacionado con fenómenos como las conductas autolesivas (Bornstein, 2012) y la violencia entre parejas (Alvarez-Dardet, Pérez Padilla \& Lorence Lara, 2013; Del Castillo, Hernandez-Díaz, Romero-Palencia \& Iglesias-Hoyos, 2015; Sierra García, Loinaz \& Echeburúa, 2012; Watson, Clark \& Tellegen, 1988).

El Cuestionario de Dependencia Emocional (CDE) es uno de los instrumentos que se ha creado con el fin de evaluarla (Lemos \& Londońo, 2006). Otros autores han propuesto cuestionarios semejantes, como el Inventario de Dependencia Emocional (Aiquipa, 2012) y el Cuestionario de Dependencia Emocional en el noviazgo (Urbiola, Estévez \& Iraurgi, 2014), en los cuales el CDE ha servido como referente teórico (Aiquipa, 2012) o como criterio de validez concurrente (Urbiola et al., 2014). Cabe anotar que anterior al CDE se habían 
creado cuestionarios que evaluaban constructos similares como el Test del Perfil Relacional (TPR), el cual evalúa la sobredependencia destructiva, el desapego disfuncional y la dependencia saludable (Bornstein et al., 2004); y el Inventario de Dependencia Interpersonal (IDI) que evalúa la confianza o dependencia emocional en la otra persona, la falta de autoconfianza social y la afirmación de autonomía (Hirschfeld et al., 1977). Sin embargo ambos cuestionarios no abordan específicamente la dependencia emocional, sino constructos similares.

$\mathrm{El} \mathrm{CDE}$ posee seis escalas que coinciden con aspectos que han sido señalados como importantes para definir a las personas con dependencia emocional y retomados en instrumentos semejantes (Lemos \& Londoño, 2006); asimismo, este cuestionario ha sido objeto de análisis psicométricos para su adaptación a otras poblaciones (Coppolino, Ingrassia, Benedetto \& Aguglia, 2015; Mendez-Zavala, Favila Figueroa, Valencia Cruz \& Diaz Loving, 2012). Cabe anotar que el estudio de Mendez-Zabala, Favila-Figueroa, Valencia-Cruz y Diaz-Loving (2012) partió de un análisis factorial exploratorio, el cual no permite confirmar si la estructura factorial original del CDE se mantenía en la población mexicana, sino que buscó una estructura factorial que explicara la mayor cantidad de varianza en la población medida; lo que llevó a que los investigadores afirmaran que se trataba de un cuestionario diferente con otra estructura factorial. Por el contrario, el estudio llevado a cabo en Italia por Coppolino, Ingrassia, Benedetto y Augulia (2015), llevó a cabo la validación del CDE mediante un análisis factorial confirmatorio, el cual mostró un buen ajuste de los factores del CDE, excepto para la expresión límite. Asimismo los índices de consistencia interna de la prueba fueron altos, la prueba total alcanzó un alfa de .93 y las subescalas entre .70 y .88, con excepción de la expresión límite que alcanzó un alfa de .49 .

Es importante anotar que el CDE ha sido utilizado en estudios orientados a la medición del perfil cognitivo (Jaller \& Lemos, 2009; Lemos et al., 2012), así como características psicopatológicas en población universitaria (Niño-Rodríguez \& Abaunza León, 2015; Siabato Macías \& Salamanca Camargo, 2015; Sierra Vargas, Güichá Duitama 
\& Salamanca Camargo, 2014). Estos estudios han evidenciado que las personas con dependencia emocional presentan creencias sobre impredecibilidad de la satisfacción de necesidades afectivas y la necesidad de evitar el malestar, a expensas de la realización personal (Jaller \& Lemos, 2009). Estas representaciones se mantienen mediante deberías relacionados con el amor romántico y una necesidad de control de su pareja, llevándolos a asumir actitudes demandantes e interpretar las rupturas de manera catastrófica (Lemos et al., 2012; Lemos, Londoño \& Zapata, 2007). Asimismo, señalan que este patrón ilustra las variables asociadas al origen de la violencia en el noviazgo, asociándose con formas de violencia verbal, chantaje, control, exigencias, violencia social, humillación, celos y conflicto (Del Castillo et al., 2015). Finalmente se ha encontrado que la dependencia emocional sería un factor fuertemente asociado con la conducta suicida en población universitaria (Siabato Macías \& Salamanca Camargo, 2015).

Cabe anotar que estos estudios parten de la base que la dependencia emocional es un factor que puede medirse con el mismo instrumento en la condición de estar o no con una pareja o en hombres y mujeres; sin embargo, esta hipótesis aún no ha sido probada, ya que ninguno de los instrumentos para medirla han sido sometidos a pruebas de invarianza métrica, de tal forma que los resultados sean limitados y presentar omisiones que afectan los resultados de la investigación sobre dependencia emocional. Contrariamente a los análisis tradicionales de varianza (e.g. ANOVA y MANOVA), en donde se realizan comparaciones entre grupos en términos de variables compuestas previa verificación de las propiedades psicométricas de las escalas de medición, a través de las pruebas de invarianza se pueden realizar comparaciones simultáneamente verificando la propiedades de las medidas utilizadas (Bollen, 1989). Cabe anotar que al realizar la validación de las propiedades psicométricas en pasos previos, no se incluyen aspectos como los errores de medición en la comparación entre grupos, lo cual puede llevar a un sesgo en los resultados de la investigación (Marsh, Morin, Parker \& Kaur, 2014). 
Una condición como el estar en pareja hace que sea más probable realizar afirmaciones acerca de la necesidad de su pareja, la modificación de planes en torno a esta y la manifestación de malestar emocional en casos de una posible ruptura (Castello, 2005). Esto teniendo presente que es esperable que la dependencia emocional sea reportada en las relaciones que son más salientes para un individuo, de tal forma que algunos autores han afirmado que los mayores niveles de dependencia en jóvenes y adultos se verían manifestados en las relaciones románticas (Darcy et al., 2005). Con respecto al sexo, se ha señalado que biológicamente las mujeres tienen mayor disposición a la empatía, la compasión y al contacto social que los hombres, lo cual es indicativo de una mayor tendencia a la vinculación afectiva (Christov-Moore et al., 2014; Luo et al., 2015; Watson et al., 1988), aspecto que se ha confirmado en otras culturas, mediante estudios que han reportado que las mujeres presentan mayor tendencia a la dependencia interpersonal que los hombres (Alonso-Arbiol, Shaver \& Yárnoz, 2002; Sanathara et al., 2003). Cabe anotar que aunque la tendencia de los estudios señala estas diferencias entre sexos, también se ha afirmado que es posible que esto sea debido a que las mujeres dan cuenta de estos comportamientos de forma explícita, mientras que en los hombres la dependencia puede manifestarse de forma implícita (Natoli \& Bornstein, 2017).

Es importante resaltar que los roles, tanto en hombres como en mujeres, forman parte de la manifestación de los comportamientos que son considerados apropiados para cada sexo según la cultura en la que el individuo se encuentra inmerso (Natoli \& Bornstein, 2017). En las culturas latinas se ha reconocido la presencia de ideas como que el hombre puede realizar conductas de dominación y control de la pareja (Falicov, 2010; Nuñez et al., 2016), las cuales contrastan con ideas del hombre caballero, orientado a las necesidades de su familia y su pareja (Estrada \& Arciniega, 2015). Asimismo frente al rol de la mujer, estudios en culturas latinas han reportado un ideal cultural de mujer que sacrifica sus propias necesidades por el bienestar de su pareja y su familia (Nuñez et al., 2016). Este parece estar asociado con los comportamientos de sumisión y auto sacrificio que reportan no solo 
mujeres mayores sino también jóvenes en Latinoamérica (MendezLuck \& Anthony, 2015). Asimismo, los comportamientos que una persona presente frente a su pareja, estarán también permeados por las creencias sobre el amor y las relaciones que esta persona tenga. Al respecto, los medios de comunicación tienen una influencia importante sobre las creencias. Se ha afirmado que la música $p o p$ presenta una idea del amor idealista, asociada con un pensamiento obsesivo y con conductas de extrema dependencia (Sussman, 2010; Vannini \& Myers, 2002). Estas creencias pueden llevar a que se facilite la presentación de la dependencia emocional en la población latina, de tal forma que se hace relevante confirmar las propiedades psicométricas de un cuestionario orientado a medir este constructo.

Todo lo anterior llevó a plantear este estudio que tuvo dos objetivos. En primera instancia, mediante la utilización de análisis estadísticos de segunda generación —modelos de ecuaciones estructurales«, pretende evaluar simultáneamente las propiedades psicométricas del CDE en grupos de personas que tienen pareja o aquellas que no cumplen esa condición; así como en mujeres y hombres. Como segundo objetivo este estudio verificó si existen diferencias en dependencia emocional entre personas con o sin pareja, y entre mujeres y hombres.

\section{Método}

Se trata de un estudio psicométrico para comprobar la invarianza métrica del Cuestionario de Dependencia Emocional entre sexos y entre personas que tienen o no pareja. El análisis de invarianza métrica llevado a cabo en este estudio permite la evaluación simultánea de las propiedades psicométricas, de tal forma que permite establecer con mayor certeza si se pueden realizar comparaciones a nivel de constructo entre los grupos en cuestión (Bollen, 1989). Este análisis se llevó a cabo con una base de datos de estudiantes universitarios seleccionados aleatoriamente en una universidad colombiana para la identificación del perfil cognitivo de dependencia emocional (Lemos et al., 2012). 


\section{Participantes}

Se contó con la participación de 569 estudiantes universitarios, de los cuales $67.8 \%$ (386) eran mujeres y $32.2 \%$ (183) eran hombres. La media de edad de los participantes fue de $19.9(D E=2.4)$ años, con edades entre los 16 y 31 años. El 59.11\% (331) de los participantes pertenecían a un estrato socioeconómico alto, seguido por un $35.71 \%$ (200) que pertenecían al estrato medio. Finalmente, el 51.2\% (290) de los estudiantes tenían pareja en el momento de la evaluación, en contraste con $48.7 \%$ (276) que no tenían). Las relaciones de pareja tenían al momento de la evaluación un promedio de duración de 10.9 meses $(D E=18.75)$. Los participantes del estudio fueron invitados a participar por auxiliares de investigación entrenadas para la evaluación. Los participantes firmaron el consentimiento informado.

\section{Medición}

Para medir la dependencia emocional se utilizó el Cuestionario de Dependencia Emocional (CDE), que es un instrumento de 23 ítems que fue construido y validado por Lemos y Londońo (2006). La prueba evalúa seis factores implicados en la dependencia emocional: Ansiedad de separación (expresiones emocionales de miedo y ansiedad ante la posibilidad de disolución de la relación) con siete ítems, expresión afectiva de la pareja (necesidad del sujeto de tener constantes expresiones de afecto que calmen su sensación de inseguridad) con cuatro ítems, modificación de planes (cambio de planes y comportamientos debido a los deseos implícitos o explícitos por satisfacer a la pareja o por la posibilidad de compartir más tiempo con ella) con cuatro ítems, miedo a la soledad (temor por no tener una relación de pareja o no sentirse amado) con tres ítems, búsqueda de atención (búsqueda activa de atención de la pareja para asegurar la permanencia en la relación y tratar de ser el centro de su vida) con dos ítems y expresión límite (expresiones impulsivas de autoagresión ante la posibilidad de una ruptura) con tres ítems (Ver tabla 1). $\mathrm{El}$ análisis factorial exploratorio realizado en el estudio original evidenció que los seis factores explicaban un $64.7 \%$ de la varianza del fenómeno. 
Los porcentajes de explicación de la varianza de cada uno de los componentes fueron de $38.9 \%$ para la ansiedad de separación, $6.3 \%$ para expresión afectiva de la pareja, 5.7\% para modificación de planes, 5.0\% para el miedo a la soledad, 4.1\% para la búsqueda de atención y 4.7\% para la expresión límite. Con respecto a la confiabilidad la prueba arrojó un alfa de Cronbach total de .93. Las subescalas presentaron alfas entre .62 (para el factor de Expresión límite) y .87 (para Ansiedad de separación).

\section{Tabla 1}

\section{Definición de las variables}

\begin{tabular}{|c|c|c|}
\hline Variable & Definición & Tipo \\
\hline \multicolumn{3}{|c|}{ Subescalas del Cuestionario de Dependencia Emocional } \\
\hline $\begin{array}{l}\text { Ansiedad de } \\
\text { separación }\end{array}$ & $\begin{array}{l}\text { Temor ante la posible ruptura (real o imaginaria) de } \\
\text { la relación o el distanciamiento de la persona con la } \\
\text { que se tiene la relación. }\end{array}$ & $\begin{array}{l}\text { Cuantitativa / } \\
\text { intervalo }\end{array}$ \\
\hline $\begin{array}{l}\text { Expresión } \\
\text { afectiva de la } \\
\text { pareja }\end{array}$ & $\begin{array}{l}\text { Necesidad de tener constantes expresiones de afecto } \\
\text { por parte de la pareja que disminuyan la sensación } \\
\text { de inseguridad. }\end{array}$ & $\begin{array}{l}\text { Cuantitativa / } \\
\text { intervalo }\end{array}$ \\
\hline $\begin{array}{l}\text { Modificación } \\
\text { de planes }\end{array}$ & $\begin{array}{l}\text { Cambio de actividades o planes con el deseo } \\
\text { explícito o implícito de satisfacer a la pareja o tener } \\
\text { más posibilidades de compartir tiempo con ella. }\end{array}$ & $\begin{array}{l}\text { Cuantitativa / } \\
\text { intervalo }\end{array}$ \\
\hline $\begin{array}{l}\text { Miedo a la } \\
\text { soledad }\end{array}$ & $\begin{array}{l}\text { Temor por no tener una pareja o no sentirse amado, } \\
\text { refleja el miedo que se tiene ante la soledad. }\end{array}$ & $\begin{array}{l}\text { Cuantitativa / } \\
\text { intervalo }\end{array}$ \\
\hline $\begin{array}{l}\text { Búsqueda de } \\
\text { atención }\end{array}$ & $\begin{array}{l}\text { Búsqueda activa de atención con el fin de tener la } \\
\text { atención exclusiva de la vida de la pareja. Puede darse } \\
\text { de forma pasiva y sumisa si es lo que la pareja desea. }\end{array}$ & $\begin{array}{l}\text { Cuantitativa / } \\
\text { intervalo }\end{array}$ \\
\hline $\begin{array}{l}\text { Expresión } \\
\text { límite }\end{array}$ & $\begin{array}{l}\text { Acciones o expresiones impulsivas orientadas } \\
\text { a la restitución de la relación asociadas con la } \\
\text { posibilidad de un posible abandono }\end{array}$ & $\begin{array}{l}\text { Cuantitativa / } \\
\text { intervalo }\end{array}$ \\
\hline \multicolumn{3}{|c|}{ Variables de análisis } \\
\hline Sexo & Condición de ser hombre o mujer & $\begin{array}{l}\text { Cualitativa / } \\
\text { nominal }\end{array}$ \\
\hline $\begin{array}{l}\text { Situación } \\
\text { sentimental }\end{array}$ & $\begin{array}{l}\text { Condición de estar o no vinculado en una relación } \\
\text { de pareja }\end{array}$ & $\begin{array}{l}\text { Cualitativa / } \\
\text { nominal }\end{array}$ \\
\hline
\end{tabular}

Fuente: Lemos y Londoño (2006). 


\section{Análisis de datos}

La validación del CDE se llevó a cabo mediante la modelación por ecuaciones estructurales (SEM; siglas en inglés) dado que el CDE fue previamente validado en una muestra colombiana utilizando el análisis factorial exploratorio. En primera instancia se ejecutó un análisis factorial confirmatorio (AFC) con la totalidad de la muestra. Contrariamente al Análisis Factorial Exploratorio, los AFC son considerados parte de la familia de análisis de ecuaciones estructurales (Harrington, 2009; Kline, 2010). La validez de constructo se evaluó a través del examen de las cargas factoriales y de las correlaciones entre los factores. En el primer caso, el valor límite es $>.4$, en el segundo $<.85$ (Brown, 2006). La fiabilidad de las escalas del AFC fue evaluada usando los coeficientes de Confiabilidad Compuesta (CC) y de Varianza Extraída Promedio (VEP); los valores límite para estos coeficientes son de .70 y .50 respectivamente (Hair, Anderson, Tatham \& Black, 1999). Una vez verificadas la validez y fiabilidad del instrumento se procedió a realizar dos AFC multigrupo (AFC-MG). Este tipo de análisis permitió verificar la invarianza métrica del instrumento en las diferentes submuestras y obtener claridad acerca de los análisis post-hoc adecuados para realizar comparaciones entre dichos grupos (de un lado, entre sexos; de otro lado participantes sin y con pareja). En el caso del presente estudio se validaron dos grados de invarianza métrica a través de dos AFC-MG. El primer AFC-MG puso a prueba la igualdad de la estructura factorial constriñendo las cargas factoriales a ser iguales a través de los grupos mediante un Modelo de Invarianza Métrica Simple (MIM-simple). Este modelo se comparó con un MIM más exigente que permite comparar los grupos con respecto de los factores o escalas (MIM-fuerte). Para la validación de ambos modelos MIM se usó el método de estimación MLR. Los modelos MIM se compararon mediante la prueba Satorra-Bentler $\left(\chi^{2 \text { diff }}\right)$ (Satorra \& Bentler, 1999). Fueron usadas las siguientes fórmulas para la realización de la prueba $\chi^{2 \text { diff }}: \mathrm{T}=\left(\mathrm{T} 0{ }^{*} \mathrm{c} 0-\mathrm{T} 1{ }^{*} \mathrm{c} 1\right) / \mathrm{cd}$, donde: $\mathrm{c} 0$ es el factor de corrección de escalamiento para el modelo 0; en este caso el modelo MIM-fuerte; 
c1 es el factor de corrección de escalamiento para el modelo 1; en este caso el modelo MIM-fuerte; d0 son los grados de libertad del modelo 0 ; $\mathrm{d} 1$ son los grados de libertad del modelo 1 ; T0 es el valor $\chi^{2 \text { diff }}$ para el modelo 0; T1 es el valor $\chi^{2 \text { diff }}$ para el modelo $1 ; \mathrm{cd}=\left(\mathrm{d} 0 *{ }^{*} \mathrm{c} 0-\mathrm{d} 1{ }^{*} \mathrm{c} 1\right) /$ $(\mathrm{d} 0-\mathrm{d} 1)$. Si la prueba $\chi^{2 \text { diff }}$ resulta significativo, se elige el modelo con menor número de constricciones, en este caso el MIM-simple pues se considera que el MIM-fuerte es una sobre-simplificación del primero. De lo contrario, se puede elegir el modelo MIM-fuerte, y por tanto se puede proceder a hacer la comparación entre grupos respecto de los factores extraídos en dicho modelo.

Para la evaluación de la adecuación de cada modelo, incluyendo el AFC inicial, se usaron los siguientes coeficientes: chi cuadrado $\chi^{2}$; el Índice de Ajuste Comparativo (CFI) y el índice Tucker-Lewis; el valor crítico para ambos coeficientes es alrededor de .95. Finalmente, para evaluar el ajuste de cada uno de los modelos, se usó el Root Mean Square Error of Approximation index (RMSEA). El valor límite pare este coeficiente es alrededor de .06 ( $\mathrm{Hu} \&$ Bentler, 1999). Todos los análisis se realizaron en el paquete estadístico Mplus (v. 6.12).

\section{Resultados}

\section{Análisis Factorial Confirmatorio Multigrupo}

Los estudiantes obtuvieron puntuaciones promedio de 58 puntos $(D E=21.4)$, con un rango entre 23 y 138 puntos en el CDE. En el AFC inicial el factor Expresión limite obtuvo un índice AVE inferior al valor límite $(<.05)$ mostrando escasa fiabilidad. Por tanto, los indicadores correspondientes a esta escala fueron eliminados del AFC inicial. Como se observa en la figura 1 se puede concluir que partiendo de los resultados del primer AFC, las escalas restantes poseen buenas propiedades psicométricas. Los reactivos tienen cargas factoriales superiores al valor crítico en cada uno de los factores correspondientes. Solo uno de los indicadores (cde23) obtuvo una varianza residual relativamente alta (.76). Sin embargo, dada su carga factorial satisfactoria se decidió 
mantenerlo como parte del instrumento. Al observar las correlaciones entre los factores, se comprueba la validez discriminante para las escalas del CDE. Adicionalmente, de acuerdo a los coeficientes CC y VEP las cinco escalas del CDE son fiables. En todos los casos, los coeficientes superaron los valores críticos para ambos índices (tabla 2).

\section{Tabla 2}

Medidas de validez convergente y confiabilidad de los factores del Cuestionario de Dependencia Emocional

\begin{tabular}{lcc}
\hline Constructo & \% VEP & CC \\
\hline Ansiedad de separación & 53 & .88 \\
Expresión afectiva de la pareja & 63 & .87 \\
Modificación de planes & 57 & .79 \\
Miedo a la soledad & 66 & .85 \\
Búsqueda de atención & 60 & .75 \\
\hline
\end{tabular}

VEP: varianza extraída promedio; CC: confiabilidad compuesta

\section{Análisis multigrupo}

Con respecto a la invarianza métrica del CDE a través de la variable sexo, la tabla 3 indica que ambos modelos MIM obtuvieron índices de ajuste satisfactorios. Con respecto al sexo, la prueba $\chi^{2 \text { diff }}$ señala que es posible aceptar el modelo MIM-fuerte. Dado que este modelo fuerza la estructura factorial, las cargas factoriales no estandarizadas y los interceptos de los indicadores a ser los mismos en cada uno de los grupos, es posible concluir que el instrumento tiene propiedades psicométricas satisfactorias e invariantes a través del sexo. Además, este resultado indica que se puede proceder a realizar la comparación entre hombres y mujeres con respecto de cada uno de los cinco factores del CDE. 


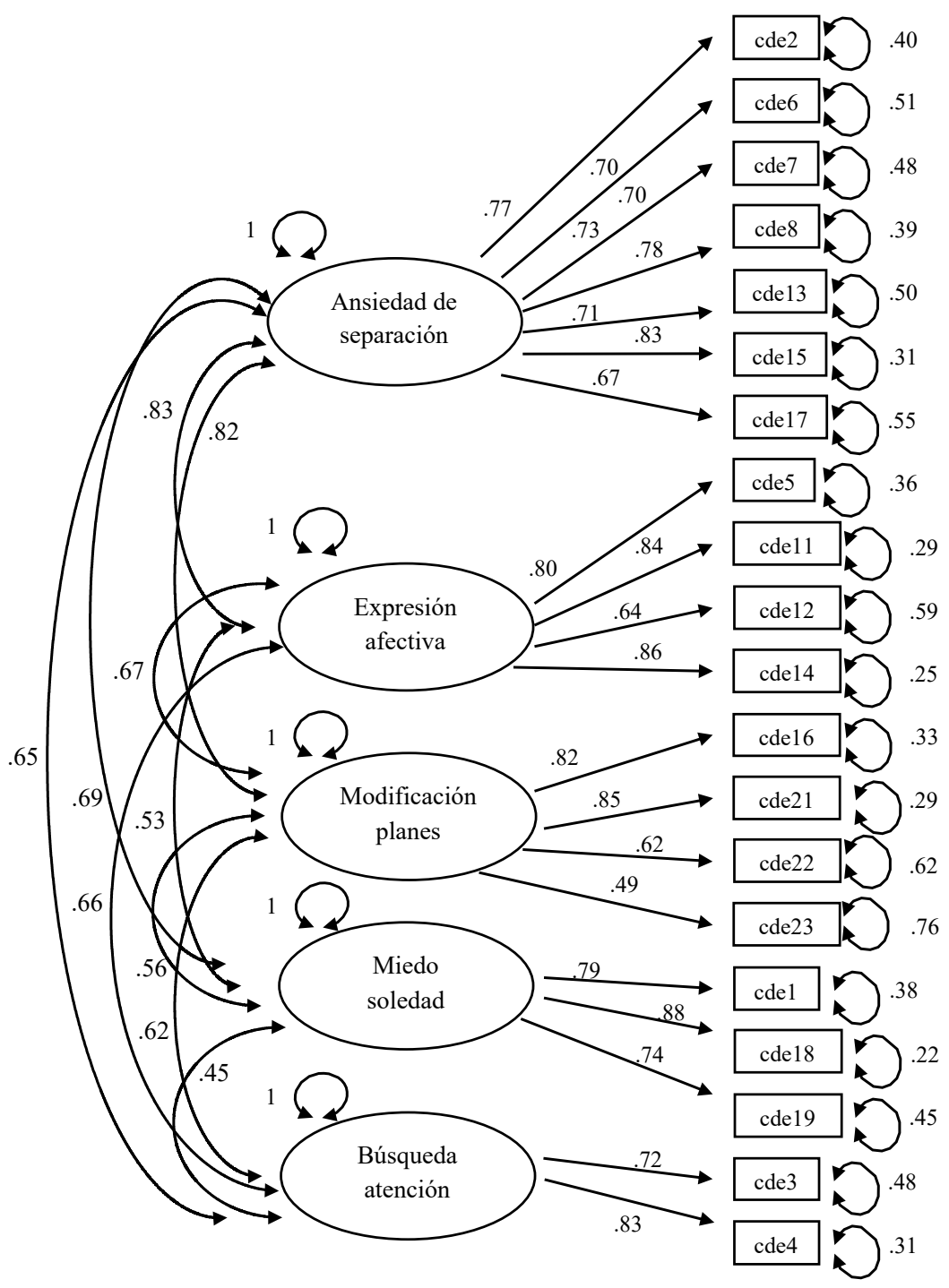

Figura 1. Análisis factorial confirmatorio Cuestionario de Dependencia Emocional (CDE) 


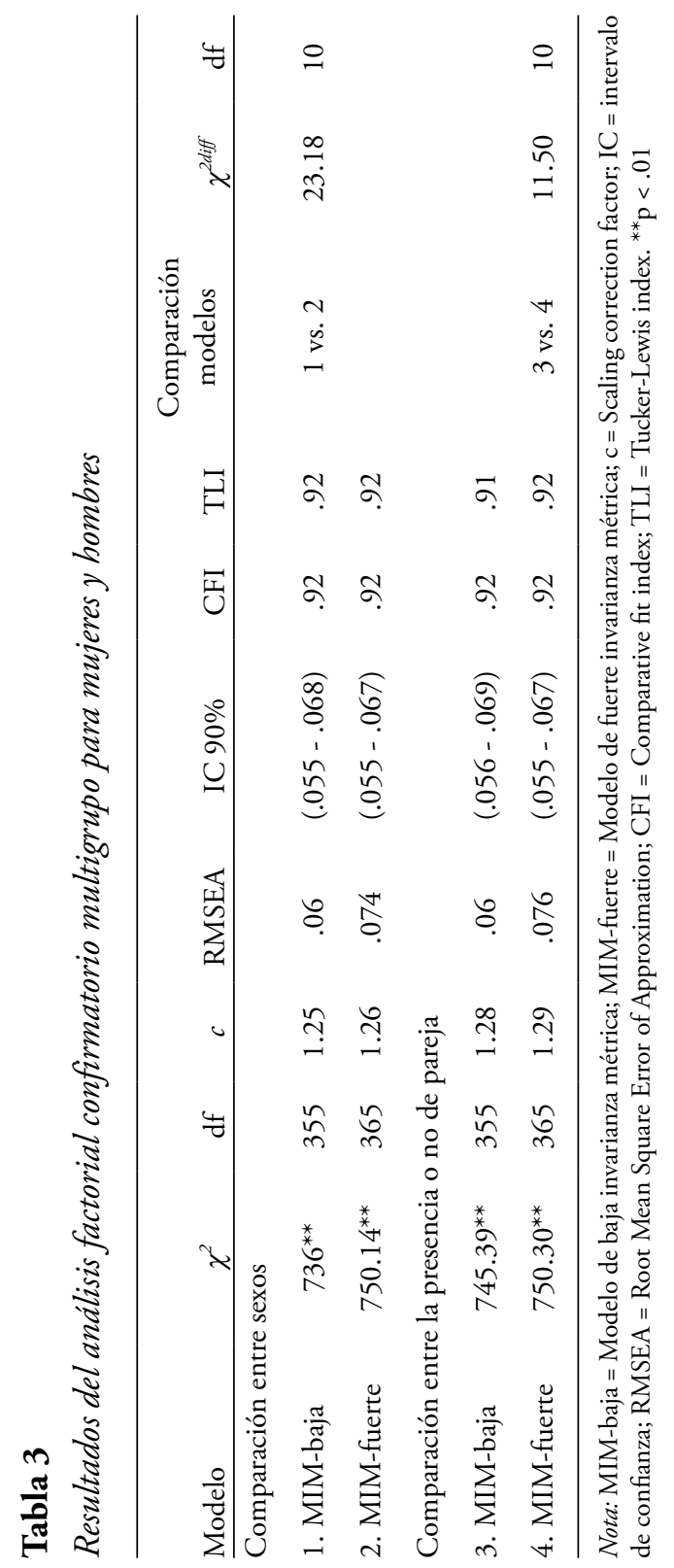


Las cargas factoriales estandarizadas del modelo MIM-fuerte se encuentran la tabla 4 . Allí se hace evidente que todas las cargas factoriales superaron el valor límite, cargan en el factor teórico correspondiente a través de los grupos, siendo en todos los casos estadísticamente significativas. A partir de estos resultados, es posible concluir que el instrumento posee validez convergente a través de los grupos conformados por mujeres de un lado, y hombres del otro.

El CDE demostró además invarianza a través de los grupos conformados por participantes con y sin pareja (tabla 3). Los coeficientes de ajuste general y el resultado de la prueba $\chi^{2 d i f f}$ indican que es posible aceptar el MIM-fuerte; por tanto, el instrumento puede ser usado para medir DE en ambos grupos pues su estructura y propiedades psicométricas no varían, además de ser útil para realizar comparaciones con respecto al constructo entre estos.

$\mathrm{Al}$ respecto, la tabla 5 permite observar la invarianza de la estructura factorial del CDE a través de los grupos de participantes con o sin pareja. Todos los reactivos cargan de manera estadísticamente significativa y por encima del valor límite en el respectivo factor teórico, lo cual indica validez convergente para el instrumento en ambos grupos.

Las correlaciones entre los constructos del CDE en ambas comparaciones fueron positivas y estadísticamente significativas, y están por debajo del valor crítico $(<.85)$ (tabla 6). Esto último sugiere que el instrumento posee validez discriminante en cada uno de los cuatro grupos considerados (mujeres, hombres, personas con y sin pareja). A propósito de la comparación entre sexos solo se encontró diferencia estadísticamente significativa en búsqueda de atención. Según los resultados de los análisis AFC-MG, los hombres puntúan más alto en esta dimensión. Con respecto a la comparación entre personas con y sin pareja, los hallazgos del estudio indican que existen diferencias estadísticamente significativas en tres de las cinco dimensiones del CDE. En resumen, las personas con pareja presentan puntajes superiores en las dimensiones ansiedad de separación, expresión afectiva de la pareja y modificación de planes. 
Invarianza del Cuestionario de Dependencia Emocional / Lemos et al.

\section{Tabla 4}

Cargas factoriales AFC-MG para mujeres y hombres

\begin{tabular}{|c|c|c|c|c|c|c|c|c|c|c|c|c|}
\hline \multicolumn{7}{|c|}{ Mujeres } & \multicolumn{6}{|c|}{ Hombres } \\
\hline Ítem & $\mathrm{F} 1$ & $F 2$ & $F 3$ & $F 4$ & $F 5$ & $R^{2}$ & $F 1$ & $F 2$ & F3 & F4 & F5 & $R^{2}$ \\
\hline 2 & .78 & & & & & .61 & .75 & & & & & .57 \\
\hline 6 & .70 & & & & & .48 & .69 & & & & & .48 \\
\hline 7 & .71 & & & & & .51 & .75 & & & & & .56 \\
\hline 8 & .77 & & & & & .60 & .81 & & & & & .66 \\
\hline 13 & .69 & & & & & .48 & .75 & & & & & .57 \\
\hline 15 & .82 & & & & & .67 & .84 & & & & & .73 \\
\hline 17 & .66 & & & & & .44 & .68 & & & & & .47 \\
\hline 5 & & .79 & & & & .63 & & .81 & & & & .66 \\
\hline 11 & & .85 & & & & .72 & & .82 & & & & .67 \\
\hline 12 & & .64 & & & & .41 & & .64 & & & & .41 \\
\hline 14 & & .87 & & & & .77 & & .84 & & & & .71 \\
\hline 16 & & & .83 & & & .69 & & & .82 & & & .67 \\
\hline 21 & & & .86 & & & .74 & & & .82 & & & .66 \\
\hline 22 & & & .61 & & & .37 & & & .65 & & & .42 \\
\hline 23 & & & .48 & & & .23 & & & .49 & & & .24 \\
\hline 1 & & & & .80 & & .64 & & & & .76 & & .59 \\
\hline 18 & & & & .88 & & .78 & & & & .88 & & .78 \\
\hline 19 & & & & .73 & & .53 & & & & .76 & & .58 \\
\hline 3 & & & & & .72 & .53 & & & & & .72 & .52 \\
\hline 4 & & & & & .83 & .68 & & & & & .82 & .69 \\
\hline
\end{tabular}

Nota: F1: Ansiedad de separación; F2: Expresión afectiva de la pareja; F3: Modificación de planes; F4: Miedo a la soledad; F5: Búsqueda de atención. Solo se reportan las cargas factoriales estandarizadas significantes al nivel $\mathrm{p}<.01$. 


\section{Tabla 5}

Cargas factoriales AFC-MG para pareja y sin pareja

\begin{tabular}{|c|c|c|c|c|c|c|c|c|c|c|c|c|}
\hline \multirow[b]{2}{*}{ Ítem } & \multicolumn{5}{|c|}{ Con Pareja } & \multicolumn{7}{|c|}{ Sin pareja } \\
\hline & $\mathrm{F} 1$ & $F 2$ & $F 3$ & $F 4$ & $F 5$ & $R^{2}$ & $F 1$ & $F 2$ & $F 3$ & F4 & $F 5$ & $R^{2}$ \\
\hline 2 & .79 & & & & & .63 & .75 & & & & & .57 \\
\hline 6 & .70 & & & & & .49 & .68 & & & & & .47 \\
\hline 7 & .77 & & & & & .60 & .68 & & & & & .46 \\
\hline 8 & .79 & & & & & .62 & .77 & & & & & .59 \\
\hline 13 & .73 & & & & & .53 & .68 & & & & & .47 \\
\hline 15 & .84 & & & & & .71 & .81 & & & & & .66 \\
\hline 17 & .70 & & & & & .49 & .63 & & & & & .40 \\
\hline 5 & & .79 & & & & .63 & & .80 & & & & .65 \\
\hline 11 & & .84 & & & & .70 & & .84 & & & & .71 \\
\hline 12 & & .63 & & & & .40 & & .64 & & & & .41 \\
\hline 14 & & .83 & & & & .75 & & .86 & & & & .74 \\
\hline 16 & & & .81 & & & .66 & & & .82 & & & .67 \\
\hline 21 & & & .86 & & & .73 & & & .83 & & & .69 \\
\hline 22 & & & .65 & & & .42 & & & .59 & & & .35 \\
\hline 23 & & & .53 & & & .28 & & & .46 & & & .21 \\
\hline 1 & & & & .77 & & .60 & & & & .79 & & .63 \\
\hline 18 & & & & .87 & & .76 & & & & .90 & & .81 \\
\hline 19 & & & & .74 & & .54 & & & & .74 & & .55 \\
\hline 3 & & & & & .72 & .52 & & & & & .73 & .53 \\
\hline 4 & & & & & .83 & .70 & & & & & .81 & .66 \\
\hline
\end{tabular}

F1: Ansiedad de separación; F2: Expresión afectiva de la pareja; F3: Modificación de planes; F4: Miedo a la soledad; F5: Búsqueda de atención. Solo se reportan las cargas factoriales estandarizadas significantes al nivel $\mathrm{p}<.01$. 


\section{Tabla 6}

Medias y correlaciones variables latentes $C D E$

\begin{tabular}{lccccccc}
\hline & \multicolumn{7}{c}{$\mathrm{M}$} \\
\hline Escala & $\mathrm{H}$ & Pareja & 1 & 2 & 3 & 4 & 5 \\
\hline 1. Ansiedad de separación & -.13 & $.32^{* *}$ & & $.83^{* *}$ & $.82^{* *}$ & $.71^{* *}$ & $.68^{* *}$ \\
2. Expresión afectiva de la pareja & -.16 & $.26^{* *}$ & $.83^{* *}$ & & $.67^{* *}$ & $.54^{* *}$ & $.68^{* *}$ \\
3. Modificación de planes & .02 & $.24^{*}$ & $.82^{* *}$ & $.67^{* *}$ & & $.56^{* *}$ & $.64^{* *}$ \\
4. Miedo a la soledad & -.18 & -.09 & $.69^{* *}$ & $.53^{* *}$ & $.56^{* *}$ & & $.45^{* *}$ \\
5. Búsqueda de atención & $.24^{*}$ & -.15 & $.66^{* *}$ & $.68^{* *}$ & $.62^{* *}$ & $.46^{* *}$ & \\
\hline
\end{tabular}

Nota: Los números en la línea horizontal se corresponden con las subescalas numeradas de forma vertical. Las correlaciones correspondientes a la comparación entre sexos fueron ubicadas en la parte inferior de la diagonal. Las correlaciones correspondientes a la comparación pareja sin pareja están situadas encima de la diagonal. ${ }^{*} p>.05 ;^{* *} p>.01 ; h=$ hombres.

\section{Discusión}

Este estudio pretendía evaluar simultáneamente las propiedades psicométricas del CDE en cuatro subgrupos de población universitaria. Los resultados del AFC seńalan que el modelo planteado por Lemos y Londoño (2006), en el que se identificó la ansiedad por separación, la expresión afectiva de la pareja, el miedo a la soledad, la búsqueda de atención y la modificación de planes como componentes de la dependencia emocional es adecuado. Sin embargo, el factor de Expresión límite que formaba parte del modelo original no mostró índices de ajuste similares de los demás factores por lo que se decidió excluirlo del modelo final. Estos resultados son similares a los reportados por Coppolino y otros en su validación de la prueba en población italiana (Coppolino et al., 2015).

Cabe anotar que la Expresión limite se ha planteado como una dimensión cognitiva y comportamental en el dependiente emocional que se presenta frente a la posible ruptura, de tal manera que se manifiesta en expresiones impulsivas similares a las del trastorno límite 
de la personalidad (Lemos \& Londoño, 2006) y en la pérdida del control de los impulsos (Castello, 2005). Esta definición evidencia el rasgo más patológico de la dependencia emocional, por lo que es posible que su validez no sea tan alta en población no clínica, y que lleve a que en análisis factoriales exploratorios se encuentre como un factor diferente al que engloba los componentes principales de la dependencia emocional como tal (Mendez-Zavala et al., 2012).

Sin embargo, debe tenerse presente que la Expresión límite, al ser el componente más extremo de la dependencia emocional, podría ser el factor que presente mayor relación con la violencia entre parejas. Esto se infiere de estudios que han mostrado la relación entre la dependencia emocional y la violencia entre parejas (Aiquipa, 2015; Bornstein, 2006b; Del Castillo et al., 2015), así como con estrategias de afrontamiento donde se ha encontrado asociación con la reacción agresiva (GonzálezJiménez \& Hernández-Romera, 2014; Niño-Rodríguez \& Abaunza León, 2015) o estudios en los que se señala que los sujetos dependientes pueden reaccionar con hostilidad cuando se enfrentan a situaciones en las que perciben un bajo control en la interacción con su pareja (Overall \& Sibley, 2009). No obstante, no se han realizado análisis que permitan verificar cuál de las dimensiones de este constructo es la que más se asocia con las expresiones violentas de una de las personas de la pareja.

Con respecto a los índices de fiabilidad encontrados en este estudio, cabe anotar que las cifras son similares a las encontradas por Lemos y Londoño (2006) en el estudio original, de tal forma que todas las subescalas presentan índices mayores a .75. Además, estos resultados son similares a los que se han encontrado en otras poblaciones donde se han reportado índices de fiabilidad de .90 y .93 para la prueba total (Coppolino et al., 2015; Mendez-Zavala et al., 2012). Los índices de confiablidad de las subescalas solo pueden compararse con los del estudio original y de la validación italiana, encontrando cifras similares (Coppolino et al., 2015; Lemos \& Londoño, 2006).

A diferencia de los estudios previos, para la validación del modelo entre personas de ambos sexos, así como en aquellos que tenían o no pareja en el momento de la evaluación, este estudio utilizó un modelo 
de ecuaciones estructurales teniendo en cuenta que la comparación entre grupos se realiza con respecto a variables latentes (dimensiones del CDE). Modelos como el ANOVA y MANOVA implican la reducción de las dimensiones a variables observadas, previa verificación de las propiedades psicométricas (Marsh, Morin, Parker \& Kaur, 2014). Por el contrario, los modelos de ecuaciones estructurales permiten realizar comparaciones entre grupos verificando simultáneamente las propiedades psicométricas de los instrumentos para todos los grupos (Bollen, 1989). A partir de esto, se pudo verificar la invarianza del constructo de la dependencia emocional y hacer afirmaciones respecto a las diferencias que se presentan entre los grupos comparados.

Anteriormente se había puesto a prueba si existen diferencias en términos de los factores del CDE entre los sexos (Coppolino et al., 2015; Lemos \& Londoño, 2006). El procedimiento utilizado entonces consistió en la evaluación de la estructura factorial del CDE y la posterior comparación de medias entre sexos. Ahora bien, las puntuaciones factoriales comparadas en test subsecuentes son consideradas poco adecuadas para la comparación de grupos en términos de constructos (Marsh et al., 2014), pudiendo llevar a resultados poco concluyentes. En este estudio, a través el test de invarianza fue posible verificar si el constructo CDE tiene el mismo significado en todos los grupos comparados. Este procedimiento, consistente en la verificación simultánea de validez de constructo en los grupos de referencia, es un paso necesario en la comparación entre grupos en términos de constructos (Dimitrov, 2010); en este caso los factores del CDE.

Así, los resultados aquí presentados con respecto a la invarianza presentada entre sexos y entre los grupos que tenían o no pareja, permite establecer que la Dependencia emocional es un constructo estable y que no depende de circunstancias ambientales. Por el contrario, está asociado con la historia del sujeto y sus vínculos afectivos, entre ellos la relación de apego tanto de su infancia como la actual (AlonsoArbiol et al., 2002; Guzmán \& Contreras, 2012; Torres-Hernández, Amador Velázquez \& Rodríguez García, 2015). Esto muestra cómo los comportamientos del dependiente emocional reflejan la visión que 
tiene de sí mismo como persona necesitada afectivamente (Guzmán \& Contreras, 2012; Karakurt, 2012; Urbiola \& Estévez, 2015), pero que no tiene la certeza de que exista otro que pueda satisfacerle esas necesidades sino que por el contrario podría ignorarlas (Bodner, Bergman \& Cohen-Fridel, 2014; Lemos et al., 2012; Lemos \& Londoño, 2006; Porter \& Chambless, 2014; Wang et al., 2014). Esto llevaría a la persona a una búsqueda de parejas emocionalmente inaccesibles, a quienes se intenta cambiar mediante la demostración del afecto y el esfuerzo por complacerlos (Ruiz-Alberto, 2013).

Con respecto a las diferencias por sexo, lo encontrado en este estudio es coherente frente al estudio original y a la validación italiana donde también se reportó mayor presencia de la búsqueda de atención en los hombres (Coppolino et al., 2015; Lemos \& Londońo, 2006). Este componente se refiere a la búsqueda activa de atención exclusiva de la pareja para asegurar su permanencia en la relación y tratar de ser el centro en la vida de este(a). Cabe anotar que no se encontraron diferencias significativas en los demás componentes de la Dependencia emocional entre sexos. Este resultado es consistente con algunos estudios previos (Antman et al., 2009; González-Jiménez \& Hernández-Romera, 2014) y arroja mayor evidencia frente a la creencia acerca de que más que una diferencia en niveles de dependencia se trata de formas particulares de manifestarla y que se vuelven válidas culturalmente. Las diferencias culturales podrían explicar que en la validación italiana sí se reportaron diferencias con respecto a dos factores adicionales, siendo mayores en mujeres, la búsqueda de expresión afectiva de la pareja y el miedo a la soledad (Coppolino et al., 2015).

$\mathrm{Al}$ respecto, cada cultura tiene modelos de pareja y de roles femenino y masculino (Brase, Adair \& Monk, 2014; Watson et al., 1988; Zandbergen \& Brown, 2015). En la cultura occidental al varón se le asigna un rol más individualista, más agresivo y competitivo, llevando a que puedan manifestar mayor búsqueda de atención (Cuddy et al., 2015; Mahalik, 2000). Por el contrario el estereotipo femenino refuerza un papel más comprensivo y de mayor tendencia a la empatía y al cuidado. De esta forma, la Dependencia emocional parecería ser 
más evidente en mujeres ya que estas podrían evidenciar más tendencia a demandar expresiones afectivas por parte de su pareja, aceptar cierto temor a la soledad o ansiedad frente a la separación, mientras que en los hombres esto los calificaría como menos masculinos (Alonso-Arbiol et al., 2002; Natoli \& Bornstein, 2017; Saldívar-Garduño et al., 2015). Esto podría llevarlos a tener manifestaciones más activas, como la búsqueda de atención, en contraste con otros factores que estarían más asociados con posiciones de sumisión y pasividad. Cabe anotar que los roles de sexo antes descritos se ven reforzados en la cultura latina por las creencias machistas y marianistas (Herrera Murcia, Nuñez Rojas, Tobón \& Arias Henao, 2009); sin embargo es posible que la diferencia encontrada entre los sexos también permita explicar el efecto que tiene el divorcio entre hombres y mujeres, en el que se ha encontrado que el impacto es mayor en hombres provenientes de culturas tradicionales colectivistas y donde el apoyo emocional se obtiene de forma primaria por parte de su pareja (Yip et al., 2012).

Con respecto a las diferencias encontradas en las personas con y sin pareja, se evidenció que las personas que se encuentran en una relación tienden a manifestar mayor ansiedad por la separación, expresión afectiva de la pareja y modificación de planes, lo que se evidencia en un mayor temor ante la separación, el distanciamiento o el abandono. Esto les genera y refuerza las pautas interpersonales de dependencia, de tal forma que se percibe a la pareja como necesaria para vivir feliz y en calma, y como la opción directa para no sentir la angustia que le genera la soledad (Lemos \& Londońo, 2006), pues tienden a pensar que la pareja podrá llenar un vacío emocional y a confundir el amor con la necesidad (Urbiola et al., 2014).

Es evidente que las manifestaciones de la Dependencia emocional son diferentes cuando las personas tienen o no pareja, ya que en el caso de no tenerla su necesidad de una relación significativa no está dirigida a una sola persona sino a la búsqueda de la misma. En el caso de las personas con pareja se evidenciarían manifestaciones comportamentales como la mayor necesidad de tener constantes expresiones de afecto de su pareja que calme la sensación de inseguridad (González-Jiménez \& 
Hernández-Romera, 2014; Lemos \& Londońo, 2006), mientras que en el caso de personas sin pareja esta demanda se vería de forma más difusa ya que lo que se evidencia es una necesidad de relaciones exclusivas con sus amigos más cercanos que los hacen ser demandantes de atención pero que no se evidenciaría estrictamente como una búsqueda de expresión afectiva (Castello, 2005). Asimismo estudios sobre apego han evidenciado que el apoyo social, que puede recibirse de otros significativos como los amigos, mediarían la relación entre la ansiedad por separación y el bienestar (Lane, 2016); de tal forma que los dependientes emocionales que no estén en una relación podrían manifestar menores niveles de ansiedad y mayores niveles de satisfacción al percibir compañía y apoyo por parte de sus amigos. Esto podría ser objeto de estudio de futuras investigaciones.

En este mismo sentido, las personas que están en una relación de pareja presentan una mayor tendencia a cambiar de actividades, planes y comportamientos debido a los deseos implícitos o explícitos por satisfacer a su pareja o a la simple posibilidad de compartir mayor tiempo con ella (Lemos \& Londoño, 2006); sin embargo las personas con dependencia emocional lo harían sobreponiendo los deseos y necesidades del ser amado por encima de los propios con la intención de agradar y manteniéndolo en primer lugar de importancia sobre otros asuntos o personas (Aiquipa, 2012; Urbiola et al., 2014). Por otra parte las personas que no tienen pareja y son dependientes emocionales tenderían a presentar comportamientos como el estar pendientes de su apariencia física, adherirse a un amigo, salir a la calle y buscar la participación en grupos con el fin de distraerse o hablar con alguien de forma más prominente que quienes no la presentan (Castello, 2005).

En síntesis, el CDE es un instrumento que muestra ser adecuado para medir la Dependencia emocional y sus componentes (ansiedad por separación, expresión afectiva de la pareja, búsqueda de atención, modificación de planes y miedo a la soledad) a través de distintos grupos poblacionales. Adicionalmente posee una escala de evaluación clínica para comportamientos extremos frente a la posible ruptura (expresión límite). 
Finalmente, y como limitaciones de este estudio, es importante señalar que para llegar a conclusiones más acertadas acerca de las diferencias en términos de dependencia emocional entre personas con y sin parejas, futuros estudios deberán realizar comparaciones intra-sujetos. Lo anterior se hace necesario puesto que el estar dentro de una relación es una condición que puede variar en el tiempo, y no una disposición biológicamente determinada como el sexo. Otra limitación es que la muestra analizada estuvo compuesta por estudiantes universitarios. Futuros estudios deberán incluir adultos en etapas posteriores del ciclo vital. Esto teniendo presente que las necesidades emocionales entre los sexos pueden variar según los desafíos de la maternidad/paternidad o el hecho de que los hijos abandonen el hogar (Bodner et al., 2014). Finalmente, este estudio se realizó con una muestra colombiana en la que asume la presencia de una ideología de los roles de sexos similar a la descrita en estudios de población latina; sin embargo, la presente investigación no evaluó el factor cultural, el cual se espera que sea abordado en futuros estudios.

\section{Referencias}

Aiquipa, J. (2012). Diseño y validación del inventario de dependencia emocional-IDE. Revista de Investigación en Psicología, 15(1), 133-145. https://doi.org/10.15381/rinvp.v15i1.3673

Aiquipa, J. (2015). Dependencia emocional en mujeres víctimas de violencia de pareja. Revista de Psicología, 33(2), 412-437. Recuperado de http://www.scielo.org.pe/pdf/psico/v33n2/a07v33n2. pdf

Alonso-Arbiol, I., Shaver, P. R. \& Yárnoz, S. (2002). Insecure attachment, gender roles, and interpersonal dependency in the Basque Country. Personal relationships, 9(4), 479-490. https://doi. org/10.1111/1475-6811.00030

Alvarez-Dardet, S. M., Pérez Padilla, J. \& Lorence Lara, B. (2013). La violencia de pareja contra la mujer en Espańa: Cuantificación y 
caracterización del problema, las víctimas, los agresores y el contexto social y profesional. Psychosocial Intervention, 22(1), 41-53. https://doi.org/10.5093/in2013a6

Antman, E. M., Selwyn, A. P., Braunwald, E. \& Loscalzo, J. (2009). Ischemic heart disease. In S. L. H. Kasper, D. Longo, J.L. Jameson \& J. Loscalzo (Eds.), Harrison's Principles of Internal Medicine (17 ed., pp. 2127-2148). EEUU: McGraw Hill.

Beck, A. T., Davis, D. D. \& Freeman, A. (2015). Cognitive therapy of personality disorders (3rd ed.). New York: Guilford Press.

Bodner, E., Bergman, Y. S. \& Cohen-Fridel, S. (2014). Do attachment styles affect the presence and search for meaning in life? Journal of Happiness Studies, 15(5), 1041-1059. https://doi.org/ 10.1007/s10902-013-9462-7

Bollen, K. A. (1989). Structural equations with latent variables. New York: Wiley.

Bornstein, R. F. (1998). Dependency in the personality disorders: Intensity, insight, expression, and defense. Journal of clinical psychology, 54(2), 175-189. https://doi.org/10.1002/(SICI)10974679(199802)54:2\&lt;175::AID-JCLP7\&gt;3.0.CO;2-R

Bornstein, R. F. (2006a). Self-schema priming and desire for test performance feedback: Further evaluation of a cognitive/interactionist model of interpersonal dependency. Self and Identity, 5(02), 110-126. https://doi.org/10.1080/15298860500492901

Bornstein, R. F. (2006b). The complex relationship between dependency and domestic violence: converging psychological factors and social forces. American Psychologist, 61(6), 595-606. https:// doi.org/10.1037/0003-066X.61.6.595

Bornstein, R. F. (2012). Illuminating a neglected clinical issue: Societal costs of interpersonal dependency and dependent personality disorder. Journal of clinical psychology, 68(7), 766-781. https:// doi.org/10.1002/jclp. 21870

Bornstein, R. F., Geiselman, K. J., Gallagher, H. A., Ng, H. M., Hughes, E. E. \& Languirand, M. A. (2004). Construct validity of the Relationship Profile Test: Impact of gender, gender role, and 
gender role stereotype. Journal of Personality Assessment, 82(1), 104-113. https://doi.org/10.1207/s15327752jpa8201_15

Brase, G. L., Adair, L. \& Monk, K. (2014). Explaining sex differences in reactions to relationship infidelities: comparisons of the roles of sex, gender, beliefs, attachment, and sociosexual orientation. Evolutionary Psychology, 12(1), 73-96. https://doi. org/10.1177/147470491401200106

Brown, T. A. (2006). Confirmatory factor analysis for applied research. New York: Guilford Publications.

Bruch, M. A., Rivet, K. M., Heimberg, R. G., Hunt, A. \& McIntosh, B. (1999). Shyness and sociotropy: Additive and interactive relations in predicting interpersonal concerns. Journal of Personality, 67(2), 373-406. https://doi.org/10.1111/1467-6494.00059

Cantazaro, A. \& Wei, M. (2010). Adult Attachment, Dependence, Self-Criticism, and Depressive Symptoms: A Test of a Mediational Model. Journal of Personality, 78(4), 1135-1162. https:// doi.org/10.1111/j.1467-6494.2010.00645.x

Castello, J. (2005). Dependencia emocional. Caracteristicas y tratamiento. Madrid: Alianza Editorial.

Christov-Moore, L., Simpson, E. A., Coudé, G., Grigaityte, K., Iacoboni, M. \& Ferrari, P. F. (2014). Empathy: gender effects in brain and behavior. Neuroscience Samp; Biobehavioral Reviews, 46(4), 604-627. https://doi.org/10.1016/j.neubiorev.2014.09.001

Coppolino, P., Ingrassia, M., Benedetto, L. \& Aguglia, E. (2015). Measuring addictive relationships in couples: Psychometric properties of the Italian version of the Cuestionario de Dependencia Emocional. Psicoterapia Cognitiva e Comportamentale, 21(3), 303-318.

Cubas-Ferreyra, D., Espinoza-Rueckner, G., Galli-Cambiaso, A. \& Terrones-Paredes, M. (2004). Intervención cognitivo-conductual en un grupo de pacientes mujeres con dependencia afectiva. Revista de psiquiatria y salud mental Hermilio Valdizan, 2, 81-99. Cuddy, A. J. C., Wolf, E. B., Glick, P., Crotty, S., Chong, J. \& Norton, M. I. (2015). Men as cultural ideals: Cultural values moderate 
gender stereotype content. Journal of personality and social psychology, 109(4), 622-635. https://doi.org/10.1037/pspi0000027

Darcy, K., Davila, J. \& Beck, J. G. (2005). Is social anxiety associated with both interpersonal avoidance and interpersonal dependence? Cognitive Therapy and Research, 29(2), 171-186. https://doi.org/10.1007/s10608-005-3163-4

Davila, J., Steinberg, S. J., Kachadourian, L., Cobb, R. \& Fincham, F. (2004). Romantic involvement and depressive symptoms in early and late adolescence: The role of a preoccupied relational style. Personal Relationships, 11(2), 161-178. https://doi. org/10.1111/j.1475-6811.2004.00076.x

Deary, I. J., Peter, A., Austin, E. \& Gibson, G. (1998). Personality traits and personality disorders. British Journal of Psychology, 89(4), 647-661. https://doi.org/10.1111/j.2044-8295.1998. tb02708.x

Del Castillo, A., Hernandez-Díaz, M. E., Romero-Palencia, A. \& Iglesias-Hoyos, S. (2015). Violencia en el noviazgo y su relación con la dependencia emocional pasiva en estudiantes universitarios. Psicumex, 5(1), 4-18.

Dimitrov, D. M. (2010). Measurement and Evaluation in Counseling and Development, 43, 121. https://doi.org/10.1177/07481756 10373459

Dunkley, D. M., Blankstein, K. R., Zuroff, D. C., Lecce, S. \& Hui, D. (2006). Neediness and connectedness and the five-factor model of personality. European Journal of Personality, 20(2), 123-136. https://doi.org/10.1002/per.578

Estrada, F. \& Arciniega, G. M. (2015). Positive masculinity among Latino men and the direct and indirect effects on well-being. Journal of Multicultural Counseling and Development, 43(3), 191-205. https://doi.org/10.1002/jmcd.12014

Falicov, C. J. (2010). Changing constructions of machismo for Latino men in therapy: "The devil never sleeps". Family Process, 49(3), 309-329. https://doi.org/10.1111/j.1545-5300.2010.01325.x 
González-Jiménez, A. J. \& Hernández-Romera, M. M. (2014). Emotional Dependency Based on the Gender of Young Adolescents in Almeria, Spain. Procedia-Social and Behavioral Sciences, 132, 527-532. https://doi.org/j.sbspro.2014.04.348 Guzmán, M. \& Contreras, P. (2012). Estilos de Apego en Relaciones de Pareja y su Asociación con la Satisfacción Marital. Psykhe, 21(1), 69-82. https://doi.org/10.4067/S0718-22282012000100005

Hair, J. F., Anderson, R. E., Tatham, R. L. \& Black, W. C. (1999). Análisis de datos multivariante. Upper Saddle River, NJ: Prentice Hall.

Harrington, D. (2009). Confirmatory Factor Analysis. New York: Oxford University Press.

Herrera Murcia, E., Nuñez Rojas, A. C., Tobón, S. \& Arias Henao, D. (2009). Análisis bibliométrico de la depresión infantil. Pensamiento Psicológico, 5(12), 59-70. Recuperado de http:// www.redalyc.org/pdf/801/80111899005.pdf

Hirschfeld, R. M. A., Klerman, G. L., Gouch, H. G., Barrett, J., Korchin, S. J. \& Chodoff, P. (1977). A measure of interpersonal dependency. Journal of personality assessment, 41(6), 610-618. https://doi.org/10.1207/s15327752jpa4106_6

Hu, L. \& Bentler, P. M. (1999). Cutoff criteria for fit indexes in covariance structure analysis: Conventional criteria versus new alternatives. Structural equation modeling: a multidisciplinary journal, 6(1), 1-55. https://doi.org/10.1080/10705519909540118

Izquierdo, S. A. \& Gómez-Acosta, A. (2013). Dependencia afectiva: Abordaje desde una perspectiva contextual. Psychologia. Avances de la disciplina, 1(7), 81-91.

Jaller, C. \& Lemos, M. (2009). Esquemas desadaptativos tempranos en estudiantes universitarios con dependencia emocional. Acta Colombiana de Psicología, 12(2), 77-83.

Karakurt, G. (2012). The interplay between self esteem, feeling of inadequacy, dependency, and romantic jealousy as a function of attachment processes among Turkish college students. 
Contemporary Family Therapy, 34(3), 334-345. https://doi. org/10.1007/s10591-012-9185-7

Kline, R. (2010). Principles and practice of structural equation modeling. New York: The Guilford Press.

Lane, J. A. (2016). Divergent Interpersonal Paths to Well-Being for Insecurely Attached Emerging Adults. Adultspan Journal, 15(2), 67-81. https://doi.org/10.1002/adsp.12022

Lemos, M., Jaller, C., González, A. M., Díaz, Z. T. \& De la Ossa, D. (2012). Perfil cognitivo de la dependencia emocional en estudiantes universitarios en Medellin, Colombia. Universitas Psychologica, 11(2), 395-404.

Lemos, M. \& Londoño, N. H. (2006). Construcción y validación del cuestionario de dependencia emocional en población colombiana. Acta colombiana de psicología, 9(2), 127-140.

Lemos, M., Londońo, N. H. \& Zapata, J. A. (2007). Distorsiones cognitivas en personas con dependencia emocional. Informes psicologicos(9), 55-69.

Luo, P., Wang, J., Jin, Y., Huang, S., Xie, M., Deng, L., . . Li, Y. (2015). Gender differences in affective sharing and selfother distinction during empathic neural responses to others' sadness. Brain imaging and behavior, 9(2), 312-322. https://doi. org/10.1007/s11682-014-9308-x

Mahalik, J. R. (2000). Gender role conflict in men as a predictor of self-ratings of behavior on the interpersonal circle. Journal of Social and Clinical Psychology, 19(2), 276-292. https://doi. org/10.1521/jscp.2000.19.2.276

Marsh, H. W., Morin, A. J., Parker, P. D. \& Kaur, G. (2014). Exploratory structural equation modeling: An integration of the best features of exploratory and confirmatory factor analysis. Annual Review of Clinical Psychology, 10, 85-110. https://doi. org/10.1146/annurev-clinpsy-032813-153700

Mendez-Luck, C. A. \& Anthony, K. P. (2015). Marianismo and caregiving role beliefs among US-born and immigrant Mexican women. Journals of Gerontology Series B: Psychological Sciences 
and Social Sciences, 71(5), 926-935. https://doi.org/10.1093/ geronb/gbv083

Mendez-Zavala, A. W., Favila Figueroa, M. A., Valencia Cruz, A. \& Diaz Loving, R. (2012). Adaptación y propiedades psicométricas del cuestionario de dependencia emocional pasiva en una muestra mexicana. Uaricha, 9(19), 113-128.

Millon, T. \& Everly, G. S. (1994). La personalidad y sus trastornos. Barcelona: Ediciones Martínez Roca.

Morse, J. Q., Robins, C. J. \& Gittes-Fox, M. (2002). Sociotropy, autonomy, and personality disorder criteria in psychiatric patients. Journal of Personality Disorders, 16(6), 549-560. https:// doi.org/10.1521/pedi.16.6.549.22140

Natoli, A. P. \& Bornstein, R. F. (2017). Integrative Assessment of Interpersonal Dependency: Contrasting Sex Differences in Response Patterns on Self-Attributed and Implicit Measures. SIS Journal of Projective Psychology \& Mental Health, 24(1), 26-33.

Nilakantan, A., Younger, J., Aron, A. \& Mackey, S. (2014). Preoccupation in an early-romantic relationship predicts experimental pain relief. Pain Medicine, 15(6), 947-953. https:// doi.org/10.1111/pme.12422

Niño-Rodríguez, D. C. \& Abaunza León, N. (2015). Relación entre dependencia emocional y afrontamiento en estudiantes universitarios. Revista Electrónica Psyconex, 7(10), 1-27.

Nuñez, A., González, P., Talavera, G. A., Sanchez-Johnsen, L., Roesch, S. C., Davis, S. M., . . Ojeda, L. (2016). Machismo, marianismo, and negative cognitive-emotional factors: Findings from the Hispanic Community Health Study/Study of Latinos Sociocultural Ancillary Study. Journal of Latinalo psychology, 4(4), 202-217. https://doi.org/10.1037/lat0000050

Overall, N. C. \& Sibley, C. G. (2009). Attachment and dependence regulation within daily interactions with romantic partners. Personal Relationships, 16(2), 239-261. https://doi.org/10.1111/ j.1475-6811.2009.01221.x 
Porter, E. \& Chambless, D. L. (2014). Shying away from a good thing: Social anxiety in romantic relationships. Journal of clinical psychology, 70(6), 546-561. https://doi.org/10.1002/jclp.22048 Roberts, R. N., Wasik, B. N., Casto, G. \& Ramey, C. T. (1991). Family Support in the Home: Programs, Policy, and Social Change. American Psychologist, 46, 131-137. https://doi. org/10.1037//0003-066x.46.2.131

Ruiz-Alberto, M. S. (2013). Dependencia emocional: características e intervención, análisis de dos casos clínicos. (Maestro en Psicología), Universidad Autónoma de Nuevo León, Monterrey, México.

Saldívar-Garduño, A., Díaz-Loving, R., Reyes-Ruiz, N. E., ArmentaHurtarte, C., López-Rosales, F., Moreno-López, M., . . . Domínguez-Guedea, M. (2015). Roles de Género y Diversidad: Validación de una Escala en Varios Contextos Culturales. Acta de Investigación Psicológica, 5(3), 2124-2147.

Sanathara, V. A., Gardner, C. O., Prescott, C. A. \& Kendler, K. S. (2003). Interpersonal dependence and major depression: Aetiological inter-relationship and gender differences. Psychological medicine, 33(05), 927-931. https://doi. org/10.1017/S0033291703007542

Santamaría, J. J., Merino, L., Montero, E., Cano, M., Fernández, T., Cubero, P., ... Gonzalez-Bueso, V. (2015). Perfil psicopatológico de pacientes con Dependencia Emocional. Cuadernos de medicina psicosomática y psiquiatria de enlace(116), 36-46.

Satorra, A. \& Bentler, P. M. (2001). A scaled difference chi-square test statistic for moment structure analysis. Psychometrika, 66(4), 507-514. https://doi.org/10.2139/ssrn.199064

Schulte, F. S., Mongrain, M. \& Flora, D. B. (2008). Healthy and unhealthy dependence: Implications for major depression. British Journal of Clinical Psychology, 47(3), 341-353. https:// doi.org/10.1348/014466508x299682

Siabato Macías, E. F. \& Salamanca Camargo, Y. (2015). Factores asociados a ideación suicida en universitarios. Psychologia. Avances de la disciplina, 9(1), 71-81. 
Sierra García, P., Loinaz, I. \& Echeburúa, E. (2012). Apego adulto en agresores de pareja. Revista de Accion Psicológica, 9(1), 33-46.

Sierra Vargas, H., Güichá Duitama, A. \& Salamanca Camargo, Y. (2014). Relación entre estilos de personalidad y estrategias de afrontamiento en estudiantes de psicología. Medicas UIS, 27(2), $15-27$.

Sussman, S. (2010). Love addiction: Definition, etiology, treatment. Sexual Addiction \& Compulsivity, 17(1), 31-45. https://doi. org/10.1080/10720161003604095

Tirapu, J., Perez, A., Calvo, R. \& Mata, I. (2005). Propuesta de un modelo dimensional para los trastornos de personalidad. Actas Españolas de Psiquiatria, 33(4), 254-262.

Torres-Hernández, A., Amador Velázquez, R. \& Rodríguez García, C. (2015). El descuido de sí misma en el proceso de Enamoramiento-Amor. Revista electrónica Medicina, salud y sociedad, 5(2), 121-139.

Urbiola, I. \& Estévez, A. (2015). Dependencia emocional y esquemas desadaptativos tempranos en el noviazgo de adolescentes y jóvenes. Psicología Conductual, 23(3), 571-587.

Urbiola, I., Estévez, A. \& Iraurgi, I. (2014). Dependencia emocional en el noviazgo de jóvenes y adolescentes (DEN): desarrollo y validación de un instrumento. Ansiedad y Estrés, 20(2-3), 101-114.

Vannini, P. \& Myers, S. M. (2002). Crazy about you: Reflections on the meanings of contemporary teen pop music. Electronic Journal of Sociology, 6(2).

Wang, S., Roche, M. J., Pincus, A. L., Conroy, D. E., Rebar, A. L. \& Ram, N. (2014). Interpersonal dependency and emotion in every day life. Journal of Research in Personality, 53, 5-12. https://doi.org/10.1016/j.jrp.2014.07.007

Watson, D., Clark, L. A. \& Tellegen, A. (1988). Development and validation of brief measures of positive and negative affect: the PANAS scales. Journal of personality and social psychology, 54(6), 1063 - 1070. https://doi.org/10.1037/0022-3514.54.6.1063 
Yip, P. S. F., Chen, Y. Y., Yousuf, S., Lee, C. K. M., Kawano, K., Routley, V., . . Clapperton, A. (2012). Towards a reassessment of the role of divorce in suicide outcomes: Evidence from five pacific rim populations. Social science bamp; medicine, 75(2), 358-366. https://doi.org/10.1016/j.socscimed.2012.03.009

Zandbergen, D. L. \& Brown, S. G. (2015). Culture and gender differences in romantic jealousy. Personality and Individual Differences, 72, 122-127. https://doi.org/10.1016/j.paid.2014. 08.035

Recibido: 21 de noviembre, 2017

Revisado: 23 de enero, 2018 Aceptado: 5 de septiembre, 2018 